\title{
A Light-Modulated Scattering Technique for Diffraction Field Measurements
}

\author{
Ayhan M. Vural and David K. Cheng \\ Contribution from the Electrical Engineering Department, Syracuse University, Syracuse, N.Y.
}

(Received October 28, 1963)

\begin{abstract}
This paper describes a scattering method for measuring microwave diffraction fields using light modulation. A small photoelectric cell is suspended in the field as a scatterer, upon which light pulses shine at an audio rate. Only the signal scattered back by the scatterer is modulated. A coherent detector is used which has an output voltage proportional to the square of the tangential component of the field under measurement. Since this method dispenses with all electrical connections to the scatterer, circuit complications and sources of error due to connection disturbance and scatterer movement are eliminated. Hitherto unavailable extensive data for the near-zone diffraction fields of finite cones are presented.
\end{abstract}

\section{Introduction}

In the study of the diffraction of electromagnetic waves from obstacles of complex shapes, an exact analytical approach is usually not possible and approximate mathematical solutions are generally remote from actual physical conditions. In these instances, experimental results obtained with the aid of carefully designed apparatus are of great importance.

The conventional probing arrangement with a trailing transmission line for measuring diffraction fields tends to distort the fields under measurement and make experimental data less reliable. This is particularly serious in phase measurements because of the unavoidable flexing in the transmission line as the probe is moved around in the field. To overcome the difficulties involved in this arrangement, a scattering technique was introduced for the measurement of electric field distributions [Justice and Rumsey, 1955]. The principle of this method is to relate the detected signal scattered back from a very short, thin, straight wire to the square of the component of the electric field along the wire. Although this system eliminates the transmission line connections, requirements of extreme frequency stability and high tuning accuracy limit the usefulness of the system from a practical point of view.

The modulated scattering technique [Richmond, 1955b] is an extension of the scattering technique which is designed to relax the tuning and stability requirements and to increase the sensitivity of the measurements by simply coding the scattered signal from a dipole. The amplitude modulation of the scattered field, which is set up by the induced currents on the scatterer, may be achieved in several ways. The original and most common method employed the nonlinear characteristic of a miniature semiconductor diode fed by an audio modulating signal. The change in the impedance of the scatterer under the modulating current causes periodic changes in the current distribution induced on the diode by the total field of interest. The amplitude of the scattered field, which is set up by these currents, is then modulated at the same rate. This modulated signal is reflected back to the source, separated from the transmitted signal by means of a hybrid junction, and subjected to coherent detection. It may be shown [Justice and Rumsey, 1955] that when the diode satisfies certain requirements, the magnitude of the detected signal is proportional to the square of the magnitude of the tangential component of the electric field along the diode and the phase is twice the phase of this component within an additive constant.

Recently this system was revised and extended to the independent measurement of magnetic field distribution [Strait and Cheng, $1962 \mathrm{a}$ and b]. The modulated scattering technique employing diode scatterers thus offers unique features of application. However, the electrical connections to the diodes carrying modulating currents still require consideration. In an experiment where it is possible or required to use a conducting ground plane, a major part of these connections may be located behind the plane. The disturbances that would be caused by the exposed parts may be reduced considerably by using cotton threads coated with a highly resistive material such as aquadag. In a more general experiment, the exposed connections would introduce practical difficulties and impair measurement accuracy. Dried aquadag coating also has a tendency to crack when the scatterer is moved around. Hence a scatterer which is electrically isolated in the measurement space with the possibility of modulation remains a desirable device. This paper describes a scattering technique using light modulation, which dispenses with connecting circuits to the scatterer entirely. 
Extensive experimental data for near-zone electric diffraction fields around finite cones will also be presented.

\section{Scattering by Light Modulation}

In the light-modulated scattering technique a small photoelectric cell is used in place of the diode scatterer. Focused pulses of a light beam shine on the cell at an audio rate. The modulating mechanism relies on the periodic change in the conductivity of the photoconductive material. Since the scatterer is now completely isolated electrically, all complications and sources of error due to connection disturbance and scatterer movement are eliminated.

\subsection{Principle of Operation}

When an obstacle is placed in the radiation zone of a source, a total field which is the sum of the incident field and the scattered field results in the space containing the source and the obstacle. To determine the diffraction field near or at the surface of the obstacle, it is necessary to measure the phase, magnitude, and polarization of electric and magnetic fields at all desired points. When a conducting scatterer is placed at some point in the total field to be measured, a current distribution is induced on the scatterer such that the field set up by the current cancels the component of the original field tangent to its surface. If the disturbance on the original field caused by the presence of the scatterer is negligible, the current on the scatterer, and hence the backscattered signal, can be related to that component of the total field along the scatterer.

The formulation of the backscattered signal due to a small photoelectric cell is essentially the same as the diode scatterer case except for the fact that the resistance change is now a function of modulating light rather than current. With an analysis using the superposition of the backscattered signals from an open-circuited dipole and from a radiating dipole, the received signal may be expressed as $[\mathrm{Hu}, 1960]$

$$
V=V_{0}-K \frac{E_{s}^{2} l_{s}^{2}}{Z+Z_{L}}
$$

where $V_{0}$ is the contribution of the open-circuited dipole, $E_{s}$ is the component of the electric field along the scatterer, $l_{s}$ is the effective length of the scatterer, $Z$ and $Z_{L}$ are the input and load impedances, respectively. $K$ is a constant if the source excitation is kept constant. Only the second term in this equation is modulated and detected because the load impedance $Z_{L}$ is the only quantity which is affected by the modulation. The magnitude of the detected signal is then proportional to the square of the magnitude of $E_{s}$, and the phase of the detected signal is equal to twice the phase of $E_{s}$ plus a constant phase shift.

\subsection{Choice of Photoconductive Material}

Photoelectric transducers are devices for converting optical impulses into electrical signals.
There are three major categories. The first class behaves primarily as variable resistors. A change in the intensity of the incident light results in a change in the conductivity of the device. These are called photoconductors. The second class produces an open-circuit voltage which is proportional to the light intensity. Finally, the response of a third class is in the form of a photocurrent proportional to the light intensity.

The photoelectric device used in this experiment belongs to the first class, since the modulation mechanism is controlled by a variation in the resistance of the scatterer. Photoconductivity is the phenomenon of an increase in the conductivity of a material when it is illuminated by light of sufficiently small wavelength. Although this effect is observed in almost all semiconductor materials and $p-n$ junctions, the magnitude and the level of this variation change from material to material. This phenomenon is simply caused by the creation of extra free carriers upon irradiation. This can occur only if the absorbed photons have sufficient energy, i.e., $h f \geq E_{g}$ where $E_{g}$ is the energy gap between the conduction and valence bands, $h$ is the Planck's constant, and $f$ is the frequency.

An extensive study of semiconductor crystals, films, and $p$ - $n$ junctions has been made to determine the characteristics appropriate for use as a modulated scatterer. Two considerations are of primary importance, namely, the operation of the devices as a dipole and the efficiency of modulation and detection. The first consideration relates primarily to the physical dimensions of the device: it must be sufficiently short, slender, and thin relative to the operating wavelength, and it is to be operated without the need of a bias. Efficiency of modulation and detection depends upon such factors as spectral response, frequency response, sensitivity, noise equivalent power and detectability, behavior at microwave frequencies, and tuning possibilities. The device should yield a maximum response at or near visible light frequencies, change its parameters with impinging light intensity when irradiated by microwaves, and offer wide resistance variations from "dark" to "light" conditions. Among the many different types of crystals, thin films, and $p-n$ junctions examined, only $\mathrm{CdSe}$ and $\mathrm{CdS}$ possess suitable microwave properties. Resistance curves for commercially available photoconductive cells CL604L and CL605L are plotted in figure 1 versus light level. A scatterer made of thin CdSe film was selected because of its low light resistance, high sensitivity, and relatively fast speed of response.

\subsection{Choice of Modulation Frequency}

Frequency response is determined by the time constant of the photoconductive material, which in turn is a function of light level. It is essentially limited by the decay time that can be directly related to the recovery time of the semiconductor material. It takes a finite time for charge carriers to recombine after the excitation has been removed.

As a more practical experiment, the amplitude of 


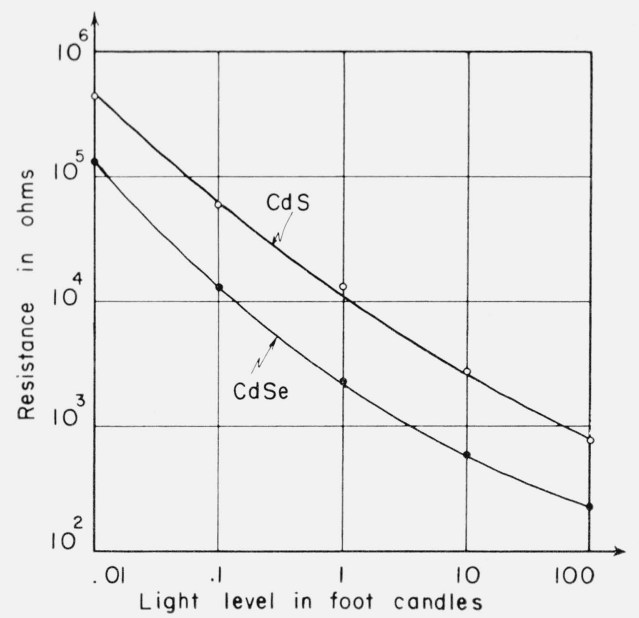

FIgURE 1. Resistance of CdS and CdSe scatterers as function of light level.

the a-c signal produced by the detector under the action of different modulation frequencies was determined. The signal was measured across a load resistor in series with a battery and the detector. Amplitude curves for CdS and CdSe detectors are shown in figure 2 as functions of modulation frequency. It is clear that in order to obtain large signal amplitudes a reasonably low modulation frequency must be used with these materials. However, high-gain amplifiers are bulky at low audiofrequencies; a modulation frequency of $300 \mathrm{c} / \mathrm{s}$ was selected as a good compromise for the overall frequency response of the system.

\subsection{The Optical System}

Once the scatterer has been selected, the performance of a photoconductive modulation system depends upon the design of a proper optical system. The optical system which was specially constructed in accordance with the needs of this experiment consists of three main parts: a light source, a chopper, and a focusing assembly.

A high-power concentrated arc lamp (type K-300) was used as the light source. A special feature of this lamp is its essentially point-source characteristic with a brightness of 29,000 candles per square inch. The high-intensity narrow beam emitted by the lamp results in a uniform, compact spot over the surface of the scatterer upon focusing. These characteristics made it possible to relax the focusing requirements to some extent. It was observed that the response of the photoconductive scatterer remained approximately constant over a distance of $\pm 5 \lambda(\lambda=3.2 \mathrm{~cm})$ about the focusing point.

Concentrated arc lamps usually do not require complicated optics. In this system focusing was accomplished by means of two sets of lenses. The condensing lens was located at the aperture of a ventilated box containing the light source. At this stage the uniform light beam emerging from the condensing lens was chopped by a rotating chopper

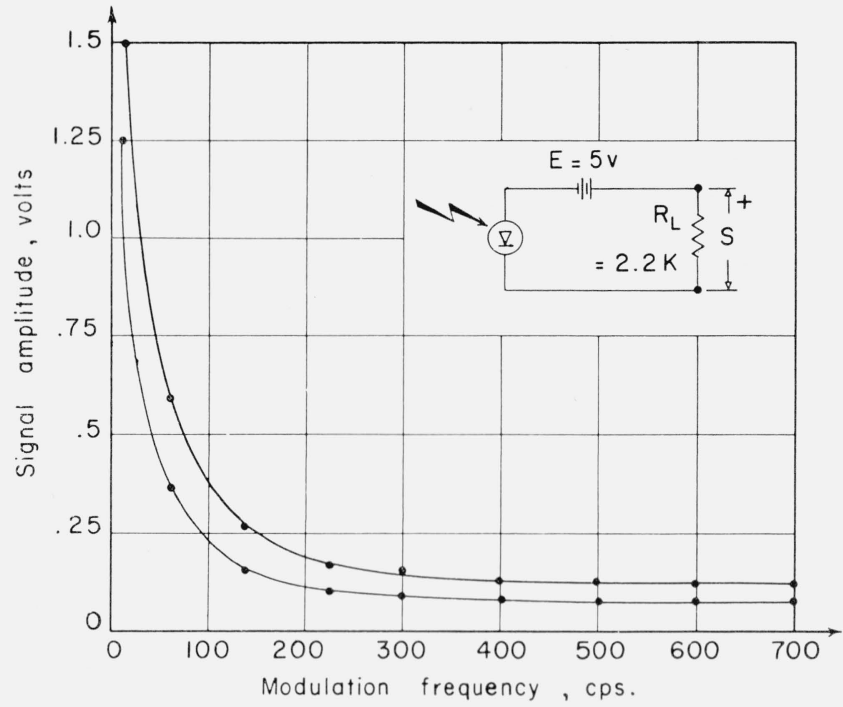

Frgure 2. Signal amplitude, S, at various chopping frequencies.

blade before it reached the focusing lens. A focusing lens of $508 \mathrm{~mm}$ focal length was used to focus the modulated light energy upon the scatterer.

The design of the chopper was based on a Fourier analysis determining the spectrum of the modulating frequency and its harmonics, and relating them to the radiation power emanating from the light source. It has been shown [McQuistan, 1959] that by this analysis a relationship between the aperture radiusto-chopper wheel radius ratio and the number of notch-tooth pairs may be established to produce a fundamental amplitude equal to the ideal sinusoidal modulation of radiation emanating from the same aperture. The same relationship holds also for the equality of the rms value of the radiation modulated in the assumed manner to the rms value of the radiation modulated in an ideal sinusoidal manner. The chopper blade was driven by a hysteresis synchronous motor operating at a fixed speed of 1800 rpm. A sequence of light impulses of frequency 300 $\mathrm{c} / \mathrm{s}$ resulted from the chopping action of the blade containing 10 notch-tooth pairs.

\section{The Experimental Setup}

The experiments were performed in an airconditioned microwave anechoic chamber. The experimental apparatus were located on a stand at one end of the chamber separated from the radiation zone by a wall of commercial electromagnetic energy absorbing material. A $20-\mathrm{db}$ horn representing the antenna was used to radiate into the room from an aperture centered on the axis of the chamber. Light illumination was obtained through another aperture in the absorbent wall. The block diagram of the apparatus used in the experiment is shown in figure 3. The use of many of the devices in the diagram is self-explanatory. The source was a V-63 klystron immersed in a large oil bath to maintain frequency 


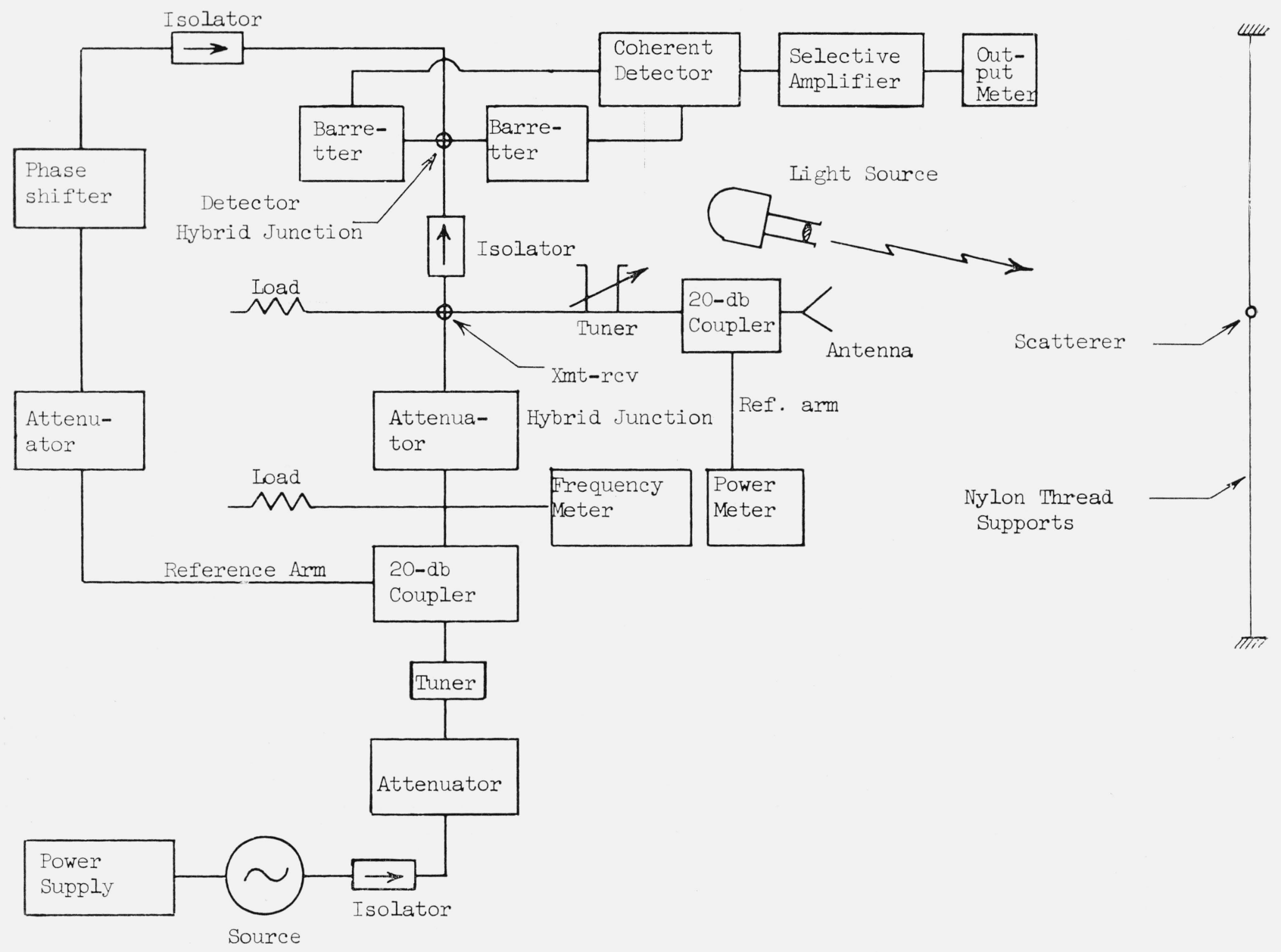

Figure 3. Block diagram of experimental set-up.

stability. The frequency of operation was 9375 $\mathrm{Mc} / \mathrm{s}(\lambda=3.2 \mathrm{~cm})$. System performance was monitored by the frequency meter and the power meter.

The source establishes an electromagnetic field through the antenna. The photoconductive scatterer modulated periodically by the light source creates a modulated scattered field which is received by the same antenna. Isolation between the transmitted and the back-scattered signals is accomplished by the properties of a transmit-receive hybrid junction. If a perfect match is obtained between the antenna and the load, the transmitted power is divided evenly between them and none enters the receiver. The received power is directed to a detector hybrid junction by the same property. This received signal and a reference signal taken from the reference arm of a 20-db coupler are then divided between the two collinear arms (in phase in one arm and out of phase in the other arm) of the detector hybrid junction in which the barretters are mounted. Some unmodulated error signal resulting from slight detuning of the transmit-receive hybrid junction will also be transferred to the detector junction. These signals are processed in a receiving system consisting of a coherent detector, a selective amplifier of $4 \mathrm{c} / \mathrm{s}$ bandwidth about $300 \mathrm{c} / \mathrm{s}$, and an output meter.

The output of the coherent detector can be shown to be [Richmond, 1955a]

$$
V=k\left|E_{0}\right|\left|E_{s}\right|^{2} \cos (2 \gamma-\beta),
$$

where $E_{0} \angle \beta$ is the reference signal, $E_{s} \angle \gamma$ is the component of $E$ along the scatterer, and $k$ is a proportionality constant. The performance of the system depends on a good tuning procedure. This procedure has been explained in detail by Strait and Cheng [1962a and b] previously. When the phase shifter in the reference arm is adjusted so that the reading of the output meter is a maximum $(\beta=2 \gamma)$, then

$$
V=k\left|E_{0}\right|\left|E_{s}\right|^{2} .
$$

Since $k$ and $E_{0}$ are constants, both the amplitude and the phase of $E_{s}$ can be determined.

The scatterer was supported by thin nylon threads attached to a large frame. The disturbance of the field due to the nonconducting supporting threads was completely negligible. The motion of the scat- 
terer through the field was accomplished by a string and pulleys arrangement attached to the frame. Since the signal scattered back from the frame is not modulated, it does not affect the accuracy of the experiment. However, the frame was constructed as large as possible in accordance with the dimensions of the room and was covered with absorbent material to minimize reflection.

To check the validity of the system, preliminary experiments were performed to map the diffraction field of a circular aperture in a conducting plane for which exact solutions exist and accurate experimental results are available [Strait and Cheng, 1962a and b]. In all cases, data obtained by using the light-modulated scattering technique checked very closely with previously published results [Vural, 1962].

\section{Diffraction Fields of Finite Cones}

Scattering of electromagnetic and acoustic waves by cones has been investigated in the literature. However, most of the previous efforts on this subject were concentrated on the evaluation of the backscattered fields in relation to the radar cross sections of semi-infinite cones. Even in this simplified problem, serious difficulties arose in evaluating numerical results from the solutions which were in terms of infinite series or integrals. When the wavelength is very short in comparison with the dimensions of the obstacle, ordinary geometric optics methods can be generalized to include diffraction rays. Keller [1960] applied the geometrical theory of diffraction to the problem of plane-wave back-scattering from a finite cone. As far as the present authors can determine, neither theoretical calculations nor experimental data are available for near-zone diffraction fields due to finite cones. The light-modulated scattering technique discussed above was adapted to measuring the diffraction fields of finite conducting cones; it was a task which could not be done with accuracy using the more conventional methods.

Measurements have been made with three right circular aluminum cones (half apex angles $20^{\circ}, 45^{\circ}$, and $67^{\circ}$ ) and with a flat aluminum disk (half apex angle $90^{\circ}$ ) of 5 -wavelength diameter. The cones have different heights, but all have a base diameter of $5 \lambda$. Because of space limitation, only selected data for the $45^{\circ}$ cone are presented here. Measurements with the disk checked very well with the published curves of Ehrlich et al. [1955]. A comparison of results is shown in figure 4.

The tip of the cone is chosen as the origin of the coordinate system, and the negative $z$-axis coincides with the axis of symmetry. The incident plane wave is polarized in the $y$-direction. Figure 5 is a plot of the square of the normalized $y$-component of the electric field, $\left|E_{y} / E_{i}\right|^{2}$, as a function of the distance from the tip of the cone along the cone axis. Distinct standing waves exist there. Figure 6 shows the distribution of $\left|E_{y} / E_{i}\right|^{2}$ at various values of $z$ in the principal $H$-plane $(y=0)$. For negative values of $z$, the readings start from points very close to, but

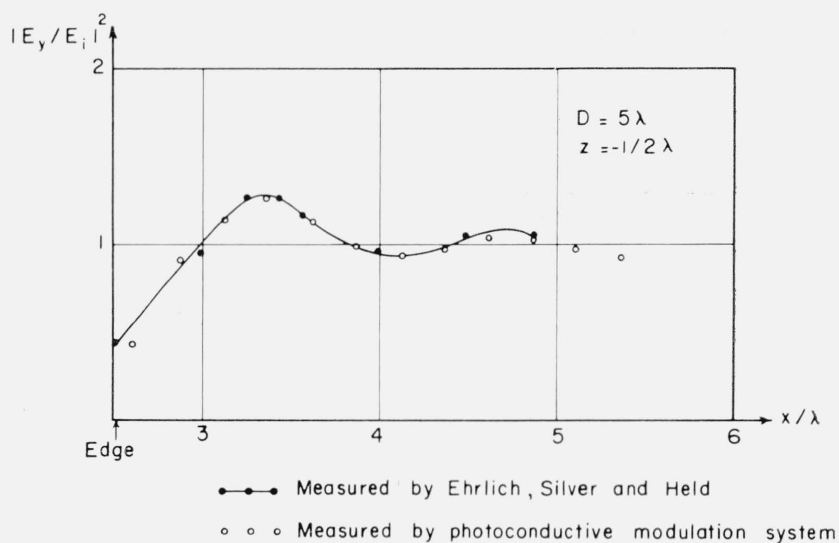

FIguRE 4. A comparison of the results obtained for the nearfield distribution of a circular disk.

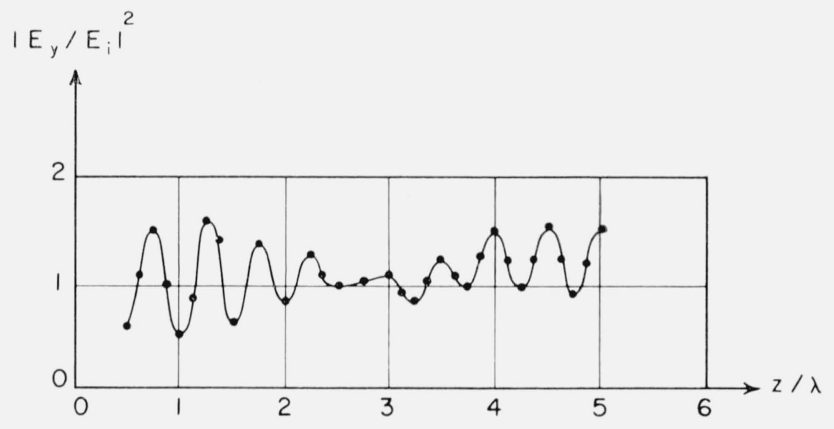

FiguRE 5. $\quad\left|\mathrm{E}_{\boldsymbol{y}} / \mathrm{Ei}\right|^{2}$ as a function of distance from cone tip for $45^{\circ}$ cone.

not quite on, the cone surface because of the finite dimensions of the photoconductive scatterer.

Fields at points off the principal $H$-plane were also measured. Sample curves in planes containing: the tip $(z=0)$ and the base $\left(z=-2 \frac{1}{2} \lambda\right)$ of the $45^{\circ}$ cone are shown in figure 7 for $y=1 \frac{1}{4} \lambda, 2 \frac{1}{2} \lambda$, and $3 \frac{3}{4} \lambda$. A composite, three-dimensional contour plot for the distribution of $\left|E_{y} / E_{i}\right|^{2}$ in the principal $H$ plane for the $45^{\circ}$ cone is given in figure 8 , which affords a better perspective. In order to show the difference in the distribution of near-zone diffraction fields in the neighborhood of finite cones of different apex angles, the corresponding contour plots of $\left|E_{y} / E_{i}\right|^{2}$ for the $20^{\circ}$ and $67^{\circ}$ cones are also given in figures 9 and 10, respectively. The cross-polarized component of the diffraction field, $E_{x} / E_{i}$, was also measured. In all cases, it was much smaller than $E / y E_{i}$.

\section{Conclusions}

A new method for measuring microwave diffraction fields has been described. It employs a small photoconductive scatterer in a light-modulated scattering technique which dispenses with connecting circuits to the scatterer entirely, thus minimizing the disturbance on the field under measurement. The varying resistance of the scatterer, changing at 

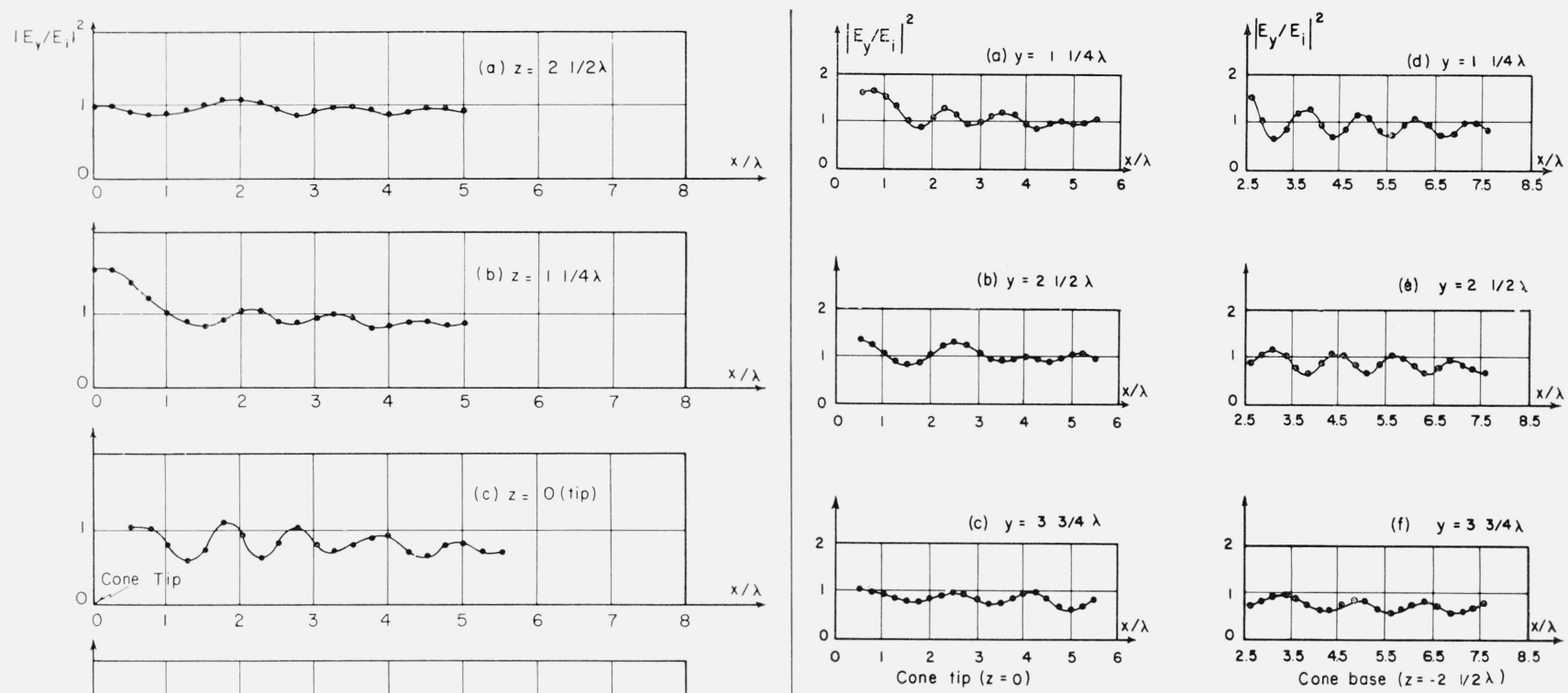

FiguRE 7. Distribution of $\left|\mathrm{E}_{\mathbf{y}} / \mathrm{Ei}\right|^{2}$ in planes parallel to principal $\mathrm{H}-$ plane $\left(45^{\circ}\right.$ cone).

Figure 6. Distribution of $\mid \mathrm{E}_{y} / \mathrm{Ei}^{2}$ at various values of $\mathrm{z}$ in principal $\mathrm{H}$-plane $(\mathrm{y}=0)$ for $45^{\circ}$ cone.

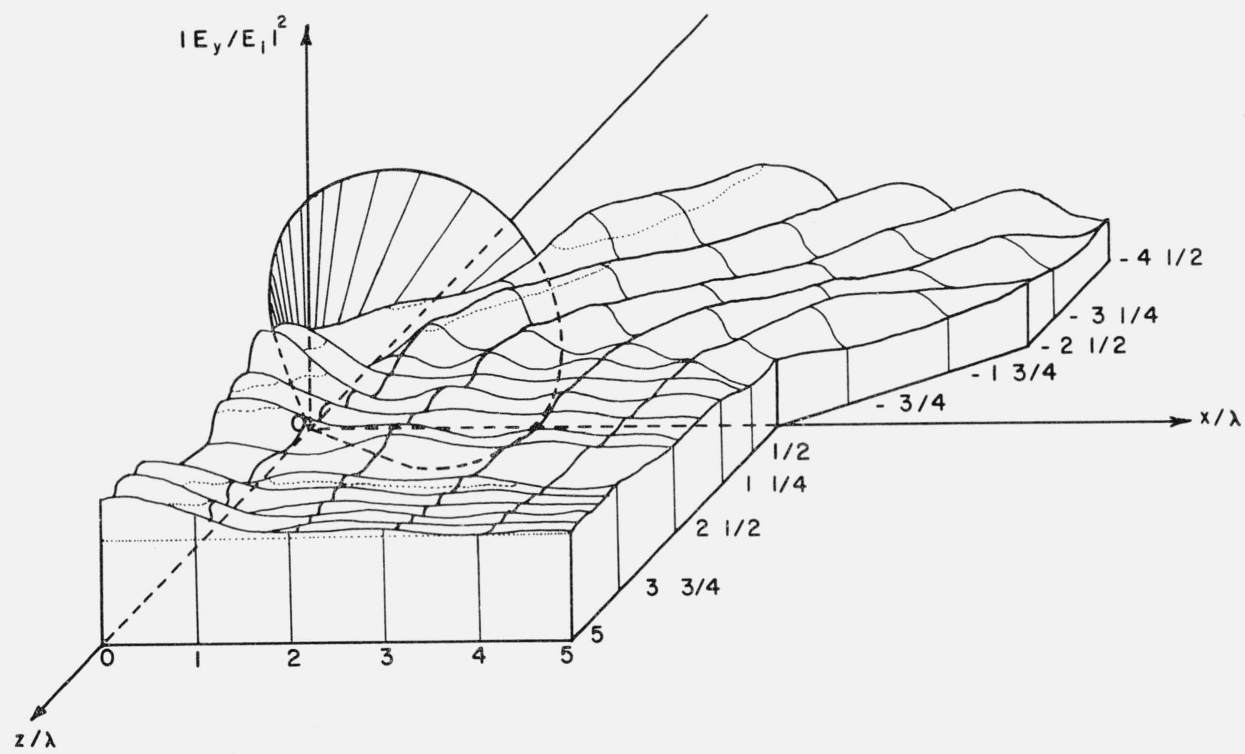

Figure 8. Three dimensional plot of $\left|\mathrm{E}_{\mathbf{y}} / \mathrm{Ei}\right|^{2}$ in principal $\mathrm{H}$-plane for $45^{\circ}$ cone. 


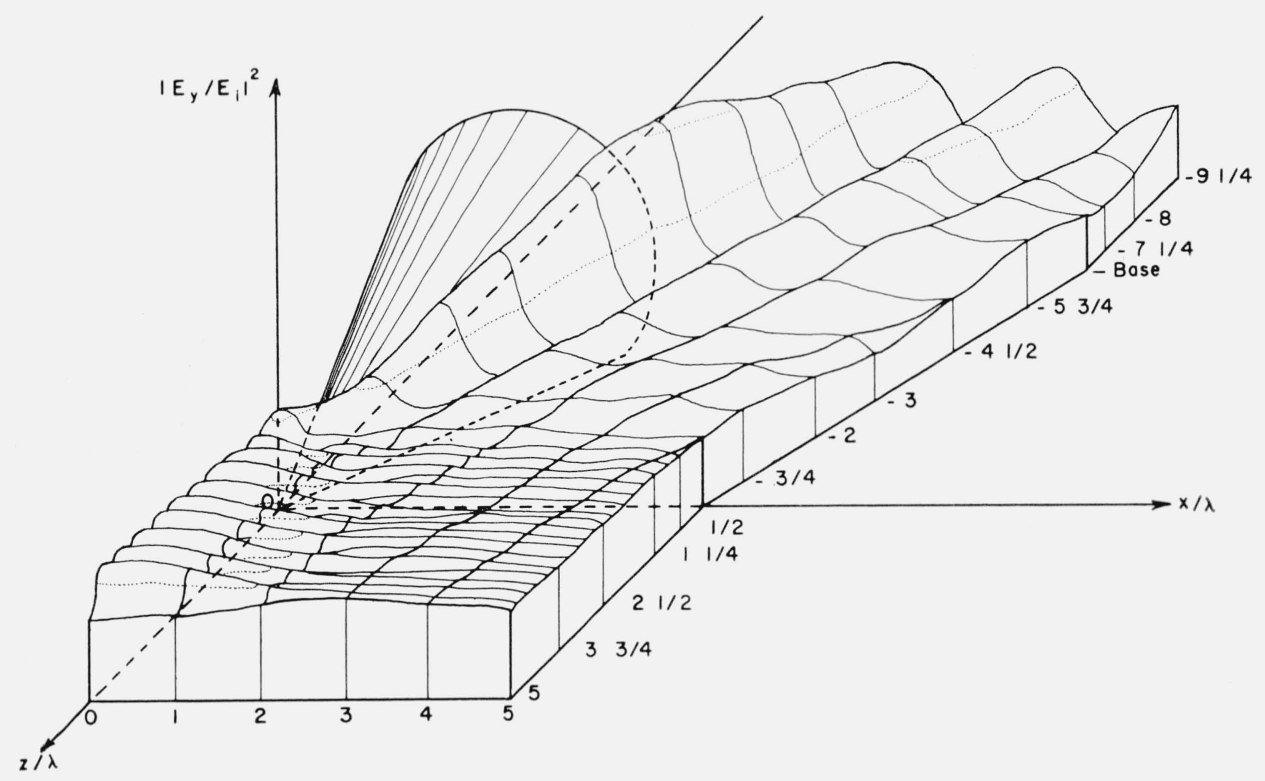

Figure 9. Three dimensional plot of $\left|\mathrm{E}_{\mathbf{y}} / \mathrm{Ei}\right|^{2}$ in the principal $\mathrm{H}$-plane for $20^{\circ}$ cone.

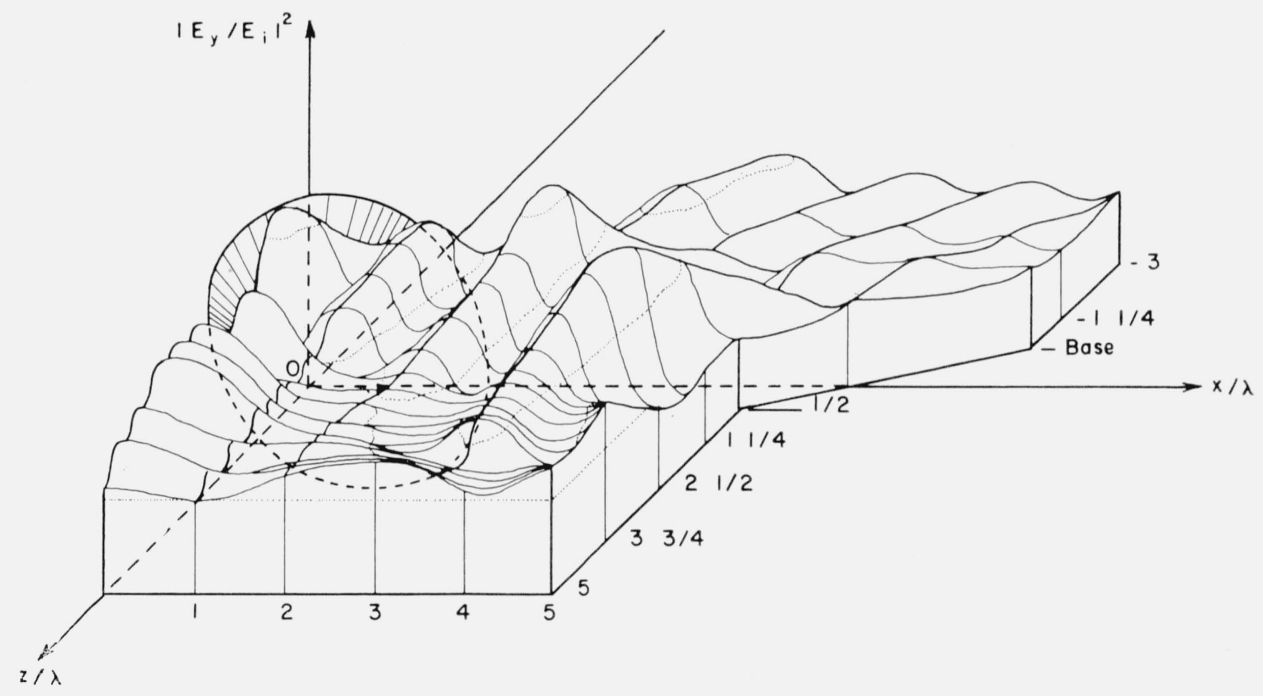

Figure 10. Three-dimensional plot of $\left|\mathrm{E}_{\mathbf{y}} / \mathrm{Ei}\right|^{2}$ in the principal $\mathrm{H}$-plane for $67^{\circ}$ cone.

the rate of the light pulses shining on it, gives rise to a received signal at the output of a coherent detecting circuit which is proportional to the square of the component of the electric field along the scatterer. Both the amplitude and the phase distributions of the diffraction field can be accurately determined. The validity of this technique has been established by comparing results with those obtained by other methods. Extensive data on near-zone diffraction fields of finite cones, which can not be measured with accuracy using more conventional methods, are presented. When sensitive photoconductive transducers with even smaller dimensions are made available in the future, the light-modulated scattering technique can also be extended for mag- netic-field measurements [Strait and Cheng, 1962a and $\mathrm{b}]$.

The authors acknowledge the valuable assistance of Bradley J. Strait during the course of this investigation. The Rome Air Development Center of the Air Force Systems Command, Griffiss Air Force Base, supported this work under Contract No. AF $30(602)-2646$.

\section{References}

Ehrlich, M. J., S. Silver, and G. Held (Mar. 1955), Studies of the diffraction of electromagnetic waves by circular apertures and complementary obstacles: The near-zone field, J. Appl. Phys. 26, No. 3, 336-345. 
Hu, M. K. (May 1960), On measurements of $E$ and $H$ field distributions by using modulated scattering methods, IRE Trans. Microwave Theory Tech. MTT-8, 295-300.

Justice, R., and V. H. Rumsey (Oct. 1955), Measurement of electric field distributions, IRE Trans. Antennas Propagation AP-3, No. 4, 177-180.

Keller, J. B. (Mar. 1960), Backscattering from a finite cone, IRE Trans. Ant. Prop. AP-8, No. 2, 175-182.

MeQuistan, R. B. (Jan. 1959), On radiation modulation, J. Opt. Soc. Am. 49, No. 1, 70-74.

Richmond, J. H. (April 1955a), Measurement of time-quadrature components of microwave signals, IRE Trans. Microwave Theory Tech. MTT-3, No. 3, 13-15.

Richmond, J. H. (July 1955b), A modulated scattering technique for measurement of field distributions, IRE Trans. Microwave Theory Tech. MTT-3, No. 4, 13-15.

Strait, B. J., and D. K. Cheng (Jan. 1962a), Microwave magnetic-field measurements by a modulated scattering technique, Proc. IEE (England) 109, Pt. B, 33-40.
Strait, B. J., and D. K. Cheng (Sept. 1962b), Measurement of microwave magnetic diffraction fields, IRE Trans. Ant. Prop. AP-10, No. 5, 643-645.

Vural, A. M. (Dec. 1962), Measurement of diffraction fields of finite cones by a scattering technique using light modulation, M.E.E. Thesis (Syracuse Univ., Syracuse, New York).

\section{Additional References}

Iizuka, K. (Oct. 1963), Photoconductive probe for measuring electromagnetic fields, Proc. IEE 110, No. 10, 1747-1754.

Vural, A. M., D. K. Cheng, and B. J. Strait (Mar. 1963), Measurement of diffraction fields of finite cones by a scattering technique using light modulation, IRE Trans. Antennas Propagation AP-11, No. 2, 200-201.

(Paper 68D4-353) 